\title{
A Cryo-FIB Lift-Out Procedure for Cryo-TEM Sample Preparation at Soft-Hard Matter Interfaces
}

\author{
K. Leifer
}

Department of Engineering Sciences, Uppsala University, Box 534, Uppsala, Sweden

TEM lamellae preparation using a focused ion beam (FIB) has become an important TEM sample preparation method in many research and development institutes. The TEM lamellae prepared with this method are typically some $10 \mathrm{~nm}$ to some $100 \mathrm{~nm}$ thick with a typical side length of some 10 microns. The preparation technique strongly relies on electron and ion beam induced deposits using Pt and $\mathrm{W}$ precursor gases. TEM lamellae for many very different materials ranging from soft to very hard materials could be successfully prepared. The FIB preparation is a unique tool for TEM lamella preparation when interfaces between hard and soft matter are to be analysed in the TEM. Using alternative methods such as conventional cross-sectional preparations or ultramicrotomy often fails for such materials.

Whereas one of the drivers of the development of highly efficient FIB based TEM lamella preparation is semiconductor industry, micro-and nanostructured devices with hard matter interfaces are now about to be developed. Examples for such interfaces are implanted biomaterials, surfaces exposed to solutions and some battery structures. The knowledge about interaction between hard matter and living cells is crucial for the development of future medical and even pharmaceutical devices.

We have developed a FIB based method to prepare TEM lamellae under cryo conditions. The particularity of this method consist in that a TEM lamella lift-out has been developed that allows to move the sample under cryogenic conditions to a TEM grid which can subsequently be transferred into a cryo-TEM holder as it is traditionally done at in the FIB lamella preparation at room-temperature. The sample can then be analysed at LN2 temperatures in the TEM. Some essential steps of the preparation method are shown in figure 1.

In this presentation we will revise the current state of cryo-FIB lamella preparation. So far, FIB milling of frozen biological specimens with subsequent TEM observation has been carried out by transferring entire pencil like samples or samples that are milled on a $\mathrm{C}-\mathrm{Cu}$ grid [1,2]. The pioneering work by Marko could, for the first time demonstrate that FIB preparation is a viable tool to locally prepare a biological sample. In this case, the sample must be grown or brought onto a TEM grid. Hayles et al. came up with a method where they successfully prepared a FIB lamella on a pencil like sample and demonstrated that amorphous ice remained stable after FIB preparation.

In the ideal picture though, one would like to scan a sample surface for spots of interest with the FIBSEM under cryo conditions and subsequently, once this position is identified, the FIB lamella should be extracted from this spot on the sample. This was the idea that governed the development of the methodology that we have developed subsequently. We rebuilt and optimized parts of the cryo stage and a transfer chamber to bring the lamella into the TEM under cryo-conditions. Thus, from the moment, where we freeze our sample until the moment of observation in the TEM, the sample can be kept at low temperature. A critical point is the temperature stability during the cryo-lift-out procedure, which is determined by the temperature of the FIB manipulator. We measured the temperature close to the tip. These temperature measurements should enable to keep low temperatures in this critical step (figure 1). 
We demonstrate the cryo-FIB lift-out, cryo-transfer and cryo-TEM observation at the example of Aspergillus niger spores that are positioned on an Al support (figure 2). After cryo-FIB milling, both, Al support and the spores are sufficiently thin for HREM, energy filtered and BF/DF imaging [3,4].

[1] M Marko et al, Nat Methods 4 (2007), p. 215.

[2] MF Hayles et al, J Struct Biol 172 (2010), p. 180.

[3] S Rubino, S Akhtar, P Melin, A Searle, P Spellward, K Leifer, J Struct Biol 180, 2012, 572.

[4] S Rubino, S Melin, P Spellward, K Leifer, (2014). J Visualized Exper: JoVE 89, 2014.

[5] We acknowledge funding from the Knut and Alice Wallenberg Stiftelse.

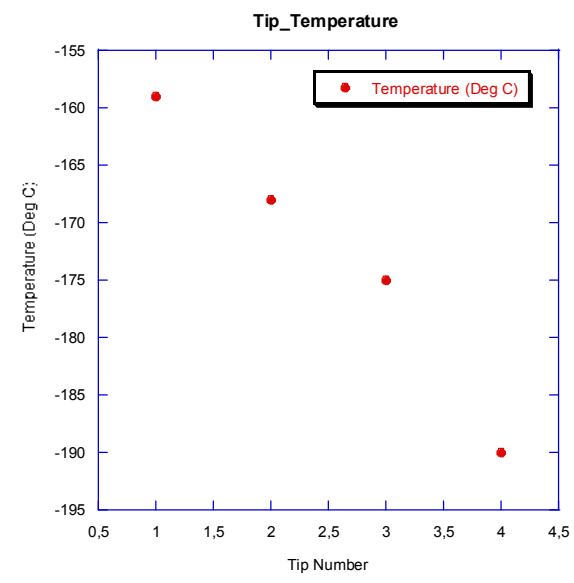

Figure 1. Temperature of the cooled FIB manipulator tip for different tips.
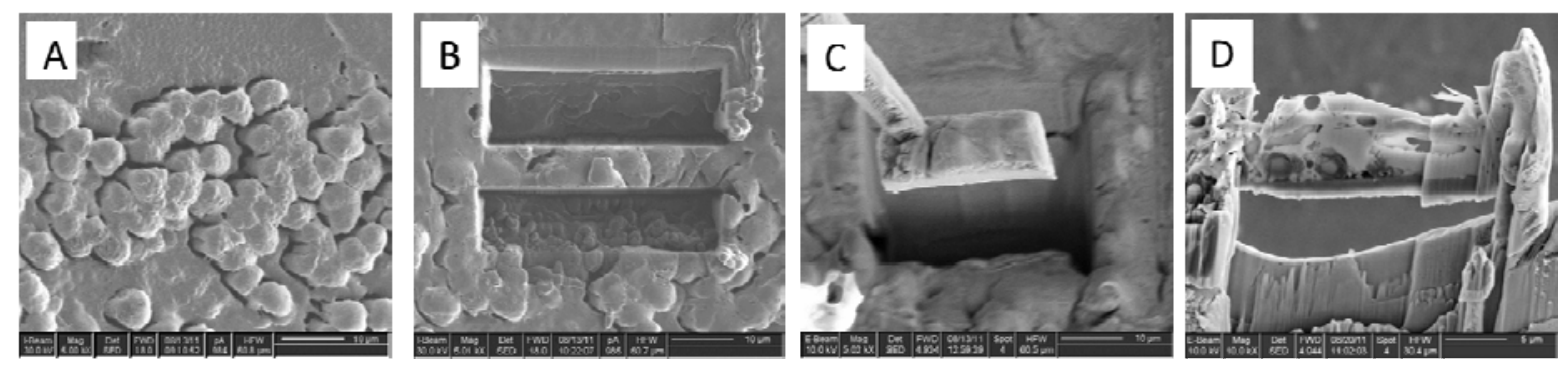

Figure 2. Some steps of cryo-FIB lamella preparation from a spore sample (A). After the cutting of the lamellae (B), the lamella is lifted out (C) and transferred to the TEM grid (D). 